\title{
Pymes madereras de Oberá (Argentina): capital intelectual y estrategia
}

\section{SMEs timber Oberá (Argentina): intellectual capital and strategy}

\author{
*Fernández Jardón, C.M. ${ }^{1}$; Martos, M.S. ${ }^{1}$; Miño Valdés, J.E. ${ }^{2}$ \\ ${ }^{1}$ Facultad de Ciencias Económicas y Empresariales de la Universidad de Vigo, \\ España \\ ${ }^{2}$ Facultad de Ingeniería, Universidad Nacional de Misiones, Argentina
}

\section{RESUMEN}

Las pequeñas y medianas empresas (pymes) son importantes para el crecimiento de las economías latinoamericanas; sin embargo, presentan limitaciones de capital intelectual, lo que puede afectar su competitividad. Este trabajo analiza el proceso de generación de ventajas competitivas en estas pymes en general y en particular, cuál es el efecto de los diferentes componentes de capital intelectual sobre el desempeño empresarial. Se define un modelo de análisis estudiando el caso de pymes madereras de Oberá (Argentina). Los resultados infieren que estas pymes utilizan el capital intelectual junto con otros recursos para generar capacidades organizativas que, unidas al territorio, afectan a sus factores estratégicos, lo que les permite obtener mejores resultados.

Palabras clave: Pymes; madera; capital intelectual; estrategia.

\begin{abstract}
Small and medium enterprises (SMEs) are important for the growth of Latin American economies; however, present limitations of intellectual capital, which may affect their competitiveness. This paper analyzes the process of generating competitive advantages in these SMEs in general and in particular, what is the effect of the different components of intellectual capital on business performance. an analysis model to study the case of SMEs timber Oberá (Argentina) is defined. The results infer that these SMEs use intellectual capital along with other resources to build organizational capabilities, coupled with the territory, affect their strategic factors, allowing them to achieve better results.
\end{abstract}

Keywords: SMEs; wood; intellectual capital; strategy.

\section{INTRODUCCIÓN}

En términos generales, la teoría del capital intelectual reconoce que existen fuertes y duraderas relaciones de negocios en el seno de sociedades con estructuras de redes, que facilitan la existencia de una lealtad perdurable de los clientes. También señalan que el papel de los empleados es clave en la empresa y que debe existir un compromiso de los empleados y empresa para la renovación y el aprendizaje (Fernández-Jardón, 2008). En las pequeñas y

\footnotetext{
*Autor Correspondiente: Fernández Jardón C.M. Facultad de Ciencias Económicas y Empresariales de la Universidad de Vigo, España.

E-mail: cjardon@uvigo.es

Fecha de recepción: setiembre 2016; Fecha de aceptación: octubre 2016.
} 
medianas empresas (pymes) es muy importante, puesto que por su tamaño deben buscar competir a través de recursos intangibles. Más específicamente, es fundamental analizar como se ve afectada la estrategia de la empresa por sus intangibles. Esta es la esencia del enfoque basado en el capital intelectual (Reed et al., 2006).

Los modelos de ventajas competitivas y capital intelectual han sido aplicados fundamentalmente en países desarrollados (Pelham, 1997; Bontis, Keow y Richardson, 2000; O’Donnell, Gilmore, Carson y Cummins, 2002; FernándezJardon y Martos, 2011). En esencia, dichos modelos sugieren que el empresario, mediante la estrategia, combina diferentes fuentes de ventajas competitivas para establecer competencias núcleo (Prahalad y Hamel, 1990), que le llevan a posicionarse mejor que sus competidores (Grant, 2005).

Giuliani et al. (2005) distinguen cuatro tipos de clústeres de empresas en Latinoamérica. Uno de los más comunes es aquel que está configurado por empresas basadas en recursos naturales, es decir, empresas cuyo input es un recurso natural o se encuentran en la cadena de valor de dicho producto. Este trabajo se centra en ese tipo de empresas. Estas pymes presentan particularidades, delimitando su competitividad, por lo que los resultados obtenidos en otros países pueden no ser válidos en este caso. Por ese motivo, tiene interés analizar el proceso de generación de ventajas competitivas para estas pymes (Fernández-Jardón y Martos, 2016). Su conocimiento permitirá desarrollar políticas y estrategias de actuación que faciliten su competitividad y, consecuentemente, potencien el desarrollo económico de los territorios donde actúan.

Diversos trabajos sostienen que el capital intelectual de la empresa puede incrementar los resultados empresariales (Bontis et al., 2000), pero no tienen en cuenta cómo se inserta ese efecto dentro del proceso mediante el cual el capital intelectual se convierte en mejor desempeño que sus competidores. El capital intelectual presenta tres componentes según el agente donde radica: el capital humano, que radica en las personas; el capital estructural, que radica en la propia organización, y el capital relacional, que radica en las relaciones que la organización tiene con el entorno (Martín-de-Castro, Navas-López, López-Sáez y Alama-Salazar, 2010). Cada uno de esos componentes puede tener un efecto específico sobre el desempeño empresarial (Fernández-Jardon y Martos, 2009). Nos interesa analizar dicho efecto dentro del proceso de generación de ventajas competitivas y determinar qué elementos del capital intelectual se integran dentro de cada componente. Ese va a ser el objetivo de este trabajo.

En particular, se va a analizar la competitividad de pymes madereras del Departamento Oberá, situado en la provincia de Misiones en Argentina, que tiene frontera con Brasil y Paraguay. Las conclusiones sugerirán proposiciones que pueden ser analizadas en otras pymes de Latinoamérica.

Para este análisis, dividiremos este artículo en varios apartados: en el primero, se establece el modelo teórico de ventajas competitivas; a continuación, se describe la situación de las empresas madereras de Oberá y la muestra seleccionada para este trabajo; después, se analizan los elementos de capital intelectual que afectan al mejor desempeño de la empresa y se definen los elementos que caracterizan el capital intelectual en la encuesta utilizada; posteriormente, se expone la metodología estadística; para continuar estudiando los resultados de la aplicación del modelo a las empresas seleccionadas; finalmente, se presenta un apartado de conclusiones.

Modelo de ventajas competitivas aplicado a pymes latinoamericanas 
Haciendo uso de los modelos de competitividad de las empresas que han estado oscilando desde una competitividad fundamentalmente externa, basada en el sector industrial (Porter, 1985), hasta una competitividad interna, basada en recursos y capacidades (Wernerfelt, 1984), de acuerdo a Grant (2005) sugerimos un modelo de ventajas competitivas, donde la combinación de recursos, mediante la estrategia, genera capacidades organizativas (factores internos ambos) que, teniendo en cuenta los factores clave de la industria (factores externos), establece las ventajas competitivas de la empresa. Por tanto, entre los factores internos, se consideran los recursos y las capacidades (Wernerfelt, 1984).

En general, se supone que los recursos son los inputs con los que cuenta la empresa para desempeñar su actividad (Grant, 1991). Los recursos pueden ser tangibles o intangibles. Los recursos tangibles incluyen los recursos financieros y los activos físicos identificados y valorados en los estados financieros de la empresa.

Los intangibles no aparecen en los estados financieros. En este grupo se integra el capital intelectual. Las pymes latinoamericanas suelen estar menos profesionalizadas (Jardón et al., 2007), con una estrategia forjada a partir de la intuición y con limitado desarrollo de las funciones empresariales (Gatto, 1999). Suelen ser individualistas (Kantis, 1996), lo que se manifiesta en los proyectos de innovación y de internacionalización realizados en solitario (Milesi et al., 2007). Esos aspectos señalan que el capital intelectual de estas pymes presenta particularidades.

Los recursos solos no son productivos. Para realizar una tarea, es necesario reunirlos en un equipo en el que deben trabajar juntos. De ahí surge la idea de capacidad organizativa, que se define como la potencialidad de una empresa de desplegar recursos para un resultado final deseado (Helfat y Lieberman, 2002). Hay una gran variedad de capacidades organizativas que, en general, necesitan la experiencia de varios individuos coordinando equipos de personas integrándolas junto con bienes de capital, tecnología y otros recursos. De acuerdo con Grant (2005), van a ser clasificadas siguiendo la cadena de valor, puesto que están orientadas a generar valor para el cliente final.

Las capacidades organizativas también presentan especiales particularidades para estas pymes; por ejemplo, la práctica directiva es más intuitiva, puesto que los gerentes de pymes suelen tener menos mentalidad estratégica, predominio de la cultura orientada a las funciones, dificultad para delegar, insuficiente profesionalización de la gestión y escaso hábito de aprendizaje continuo (Camisón-Zornoza, 1997). Sin embargo, el hecho de que en los directivos recaigan la mayoría de las decisiones en materia de gestión, les confiere a estos un profundo conocimiento del negocio que agiliza la toma de decisiones (Camisón-Zornoza, 1997). Sus limitaciones tecnológicas (Zevallos, 2006) y su escasa profesionalización (Jardon et al., 2007) les lleva a desarrollar procesos menos organizados, dificultando la introducción de rutinas organizativas en la empresa. Sin embargo, estas particularidades no parecen afectar a la definición de capacidades organizativas, que necesitan estar formadas partir de los recursos.

Todas las consideraciones anteriores implican reajustes en el modelo de ventajas competitivas. Partimos de que los recursos, al ser combinados, generan las capacidades organizativas, coincidiendo con el modelo de países desarrollados. Sin embargo, estas pymes mezclan los conceptos de estrategia y factores clave de la actividad, asociándolos a sus ventajas competitivas. Por consiguiente, tanto el territorio como las capacidades organizativas afectan a los 
factores estratégicos, que son los que generan mejor desempeño empresarial. De esa forma, las ventajas competitivas de estas pymes se difuminan dentro de sus factores estratégicos. Dentro de ese proceso, analizaremos el efecto específico de cada dimensión del capital intelectual.

La Figura 1 recoge el proceso conjunto de obtención de resultados empresariales en este tipo de empresas. Dentro de los recursos intangibles se diferencian los componentes de capital intelectual que van a ser analizadas en el caso de las empresas madereras de Oberá (Misiones, Argentina).

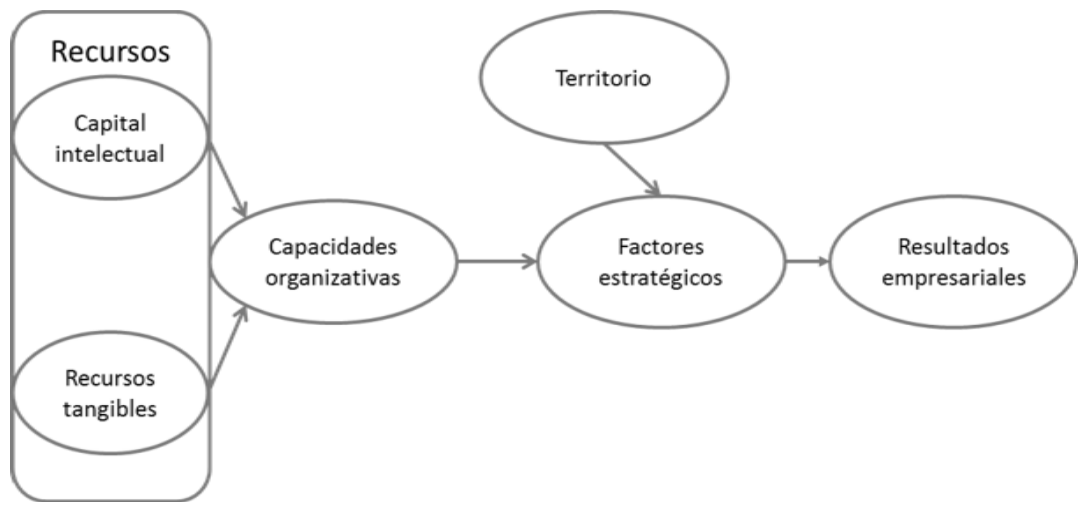

Figura 1. Relación entre factores de competitividad y resultados Fuente: Elaboración propia

\section{Las pymes madereras de Oberá}

El Departamento Oberá se sitúa en la Zona Centro Este de la Provincia de Misiones (Argentina), con una superficie de $1.620 \mathrm{~km}^{2}$, que viene a ser el 5,2\% del total de la provincia. La población del departamento es el 9,9\% de la población total provincial teniendo aproximadamente el $38 \%$ de población rural (INDEC, 2002). Dada la tradición de inmigrantes, la zona presenta una herencia nativa y europea. Su producción está orientada al sector agroindustrial puesto que el clima favorece la explotación de cultivos como té, yerba mate y tabaco que constituyen el $57 \%$ del total cultivado. También es muy importante el sector foresto-industrial, destacando la actividad de bosques tanto nativos como implantados. A partir de la crisis del 2001, esta actividad se ha desarrollado en la zona con grandes variaciones en el tipo de empresas (Escobar, 2004), si bien la mayoría son empresas muy pequeñas, que se dedican al aserrado, tanto de madera nativa como de implantados.

Dicho Departamento contaba con 162 empresas de la actividad maderera, repartidas de la siguiente manera: Oberá $(33 \%)$, Panambí $(21 \%)$, Campo Viera $(11 \%)$, Los Helechos y Campo Ramón (9\%), Guaraní (8\%), Alvear y General Alberdi (4\%) y San Martin (1\%). Aunque la mayor parte de la madera utilizada proviene de maderas implantadas $\left(23.856,04 \mathrm{~m}^{3}\right)$, también se hace uso de maderas nativas $\left(3.649,20 \mathrm{~m}^{3}\right.$ ) (MEyRNRT, 2003). El consumo aparente de rollizos de bosques implantados está destinado a madera aserrada (96\%), para tableros $(1 \%)$ y para remanufactura $(2,5 \%)$, quedando una parte reducida para muebles y fabricación de casas y aberturas. Por otro lado, los sectores con producción de capital intensivo, orientada al sector externo, han tenido una gran expansión en los últimos años. 
La tecnología utilizada era básicamente importada, con excepción de unas pocas empresas fabricantes de bienes de capital y herramientas (Maslatón y Ladrón Gonzalez, 2008). Estas últimas, aunque han tenido cierto desarrollo debido a los efectos de la apertura económica que hubo en Argentina en los noventa, tuvieron después dificultades para seguir el ritmo de los avances tecnológicos. Abastecen mercados locales de pymes y, en menor medida, han avanzado hacia la exportación luego de la devaluación, con preponderancia hacia países limítrofes. Las empresas apenas acuden a servicios de apoyo, salvo para aspectos contables y legales. En el sector privado, la Investigación, desarrollo e innovación es casi inexistente, con excepción de algunos desarrollos realizados por grandes empresas de zonas cercanas en campos como genética, técnicas de manejo y servicios forestales (Bercovich et al., 2003).

La información para el estudio se obtuvo mediante encuestas realizadas en aserraderos de madera y carpinterías localizados en el Departamento Oberá, durante finales de 2005 y principios de 2006. Se diseñó una muestra aleatoria de 120 empresas representativa de cada uno de los municipios del departamento, con un error de muestreo del $5 \%$ a un nivel de confianza del $95 \%$, suponiendo una varianza máxima donde $p=q=0,5$. A cada empresa, se le entregó personalmente o vía mail una copia resumida del proyecto original, con una breve explicación sobre la forma de cumplimentar los cuestionarios y se pasó a retirarlos una o dos semanas después, según se acordara, resolviendo las dificultades que aún quedaban pendientes. La tasa de respuesta fue del $94 \%$. Tras el proceso de depuración, quedaron 98 encuestas válidas. Se comprobó que la estructura final de la muestra se ajustaba a la población de estudio mediante un test de homogeneidad de poblaciones (Newbold, Carlson y Thorne, 2002). El cuestionario se llevó a cabo utilizando las medidas señaladas en Fernández-Jardón y Martos (2016)

\section{El capital intelectual en pymes Latinoamericanas}

Definimos el capital intelectual como el conjunto de activos intangibles que permiten generar valor para el cliente final (Bontis, 2001). Por lo que parece lógico aceptar que el capital intelectual sea fuente de ventajas competitivas (Bontis et al., 2000; Wang y Chang, 2005; Fernández-Jardon y Martos, 2009). Trabajos previos han estudiado diferentes elementos del capital intelectual como fuente de ventaja competitiva (Flöstrand, 2006; Kamoche, 1996), pero es menos común analizarlo de forma conjunta (Kamukama et al., 2011), utilizando estas como mediador de su efecto sobre los resultados.

La generalidad de los autores admiten que el capital intelectual está dividido en diferentes componentes, según el sujeto en el que se encuentra dicho capital. Por tanto, parece conveniente analizar de modo separado si existe impacto de cada uno de esos componentes sobre los resultados empresariales. Aunque aún no hay consenso en la literatura sobre las dimensiones del capital intelectual, se suelen tener en cuenta tres dimensiones (Roos et al., 1997): capital humano, que es el conjunto de valores, actitudes, aptitudes y capacidades, de los empleados que permite generar valor a la empresa (Bontis et al., 2000); capital estructural, "aquel conocimiento que la empresa internaliza (generando valor para ella) y que permanece en la organización aun cuando sus empleados se marchan a sus casas por la noche" (Roos et al., 1997, p. 42); capital relacional, definido como el valor que para una empresa tiene el conjunto de relaciones que mantiene con el entorno y con los proveedores, los competidores y los clientes (Bontis et al., 2000). 
Por consiguiente, vamos a analizar cada una de esas dimensiones y sus relaciones con los resultados de la empresa. El capital humano presenta diferencias respecto a la gran empresa, puesto que el pequeño tamaño de la pyme permite una gran proximidad entre los trabajadores que mejora la comunicación y, por consiguiente, el clima de trabajo (Wilkinson, 1999). Estas diferencias se acentúan en pymes latinoamericanas. El capital humano radica en los recursos humanos, por lo que se espera que estos sean fuentes de ventajas competitivas (Lawler, 2009). Siguiendo el modelo de gestión de competencias (Spencer y Spencer, 1993), el capital humano también incluye la actitud, la formación y las capacidades de los trabajadores y directivos. La actitud de los trabajadores y directivos va a influir en la forma de realizar su trabajo y, por consiguiente, en la calidad de los productos y servicios (Kamoche, 1996). Cuanta mayor formación y conocimientos tenga el personal de la empresa, mayor número de actividades pueden realizar, mejor se adaptan a los cambios de entorno y con más perfección realizan sus tareas (Yoguel, 2000). En las pymes basadas en recursos naturales son especialmente destacables las habilidades: a) de innovación, que sirve para desarrollar nuevas formas de capacidades organizativas o mejora en las existentes, permitiendo desarrollar nuevos procesos, sistemas y productos, y b) la de evaluación de riesgos, que ayuda a reducir los costes de financiación, que son esenciales en estas pymes. También lo son aquellas que permiten continuidad en el proceso de generar capacidades organizativas, como la habilidad para transmitir experiencias.

Todos estos elementos generan mejores capacidades organizativas, puesto que los recursos humanos intervienen en las primeras siempre que alguno de los segundos se incluya en el paquete que está asociado a la capacidad. Ese recurso lleva incluido su capital humano.

Es habitual tener en cuenta como fuente de ventaja competitiva elementos relacionados con el capital estructural, tales como la cultura empresarial que resume el conjunto de los valores existentes en la empresa (Barney, 1996). Una cultura orientada hacia la rentabilidad y a generar valor para el cliente final facilita que la empresa obtenga esa rentabilidad y, por consiguiente, que la empresa tenga éxito. La limitada profesionalización de las pymes latinoamericanas hace que la cultura esté asociada a los valores del gerente o del fundador, por lo que sus características personales condicionan la competitividad de la empresa. Evidentemente, las capacidades que organiza la empresa van a estar imbuidas de esos aspectos culturales. También es un factor importante el sistema organizativo (Martín-de-Castro et al., 2006). La organización de estas empresas suele ser desestructurada, por lo que es conveniente buscar sistemas de organización que favorezcan el desarrollo de procesos con esas características. Por ejemplo, el trabajo en equipo (Coff, 2008) ayuda a generar mejores resultados y a ser más eficientes. La existencia de una buena comunicación interna también favorece el desarrollo de capacidades en ese entorno, puesto que la organización se suele dar de modo informal. En Latinoamérica, la tecnología es un factor de diferenciación esencial, pero estas pymes presentan una tecnología obsoleta 0 inexistente, con limitados conocimientos por parte de sus usuarios (Zevallos, 2006). Eso hace más esencial el conocimiento tecnológico (Tsai, 2004). Las empresas basadas en recursos naturales tienden a estar orientadas a los productos, descuidando su orientación al marketing. Por ello, para generar valor para el cliente, es necesario un mejor conocimiento del mercado.

Las capacidades necesarias para llevar a cabo los procesos que generan valor para el cliente se apoyan en los elementos anteriormente enumerados, puesto 
que la organización permite estructurarlos, la cultura, imbuirlos y el conocimiento se introduce en cada uno de ellos.

El capital relacional es un elemento clave para competir en cualquier empresa. En lo relativo a las relaciones con clientes, destaca su lealtad hacia la organización, la confianza en las relaciones, los canales de distribución, así como las ventas repetidas y cruzadas (Flostrand, 2006); todo ello mejora el éxito competitivo. De igual forma, las relaciones con proveedores, fundamentadas en la estabilidad, confianza y compromiso, tienen efectos muy beneficiosos en la gestión de la cadena de suministros (Yang, 2008). De modo global, la constitución de redes de colaboración y alianzas fomentan las innovaciones tecnológicas (Welbourne y Pardo del Val, 2008) y es una fuente importante de aprendizaje organizativo. Las pymes latinoamericanas no suelen participar en consorcios y asociaciones y tienden a actuar individualmente (Gatto, 1999), por lo que la cooperación se vuelve mucho más necesaria. La cooperación facilita encontrar los elementos que la empresa necesita para hacer frente a las dificultades del entorno (Humble, Jackson y Thomson, 1994).

Estas empresas no se preocupan por su imagen en el momento inicial, pero suelen buscar un cierto reconocimiento social (Toledo-López et al., 2012) en su entorno local, lo que le permite añadir un cierto valor a sus productos, especialmente para sus grupos de interés (Flostrand, 2006). Estos elementos de capital relacional resultan de gran utilidad para la empresa, puesto que ofrece una valoración externa en su base de conocimientos actuales y brinda información acerca de las tendencias o intereses que muestran los agentes de su entorno, los cuales resultan cruciales para detectar oportunidades que guíen su proceso de desarrollo de nuevos conocimientos (Welbourne y Pardo del Val, 2008).

La Figura 2 recoge el impacto que interesa analizar en el modelo, centrándose esencialmente en el efecto que cada componente del capital intelectual produce sobre el proceso de generación de resultados empresariales.

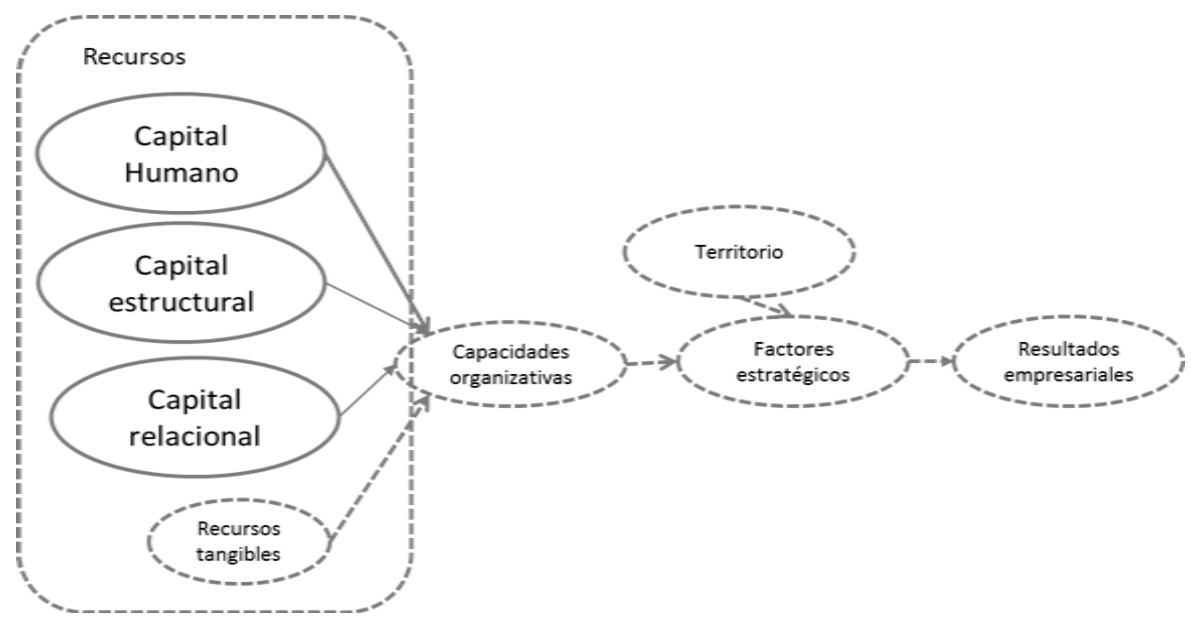

Figura 2. El capital intelectual como fuente de ventaja competitiva Fuente: Elaboración propia 


\section{MATERIALES Y MÉTODOS}

Para analizar el modelo, agrupamos las fuentes de ventajas competitivas en variables estructurales de acuerdo al factor que representan y buscamos las relaciones entre ellas. Hacemos uso de una estimación directa de esas relaciones mediante técnicas PLS (Chin, 1998), puesto que los modelos basados en covarianzas exigen suposiciones muy exigentes, especialmente en el comportamiento normal conjunto de las variables utilizadas en el modelo. El hecho de trabajar con datos ordinales medidos en una escala de intervalo, al estar basados en encuestas según la escala de Likert, se hace difícil que se verifique la suposición de normalidad de las variables. La técnica PLS es más flexible. En cada paso, intenta minimizar la varianza residual con respecto a un subconjunto de parámetros estimados, fijadas las variables de aproximación restantes y los otros parámetros.

Dicha técnica utiliza la varianza promedio extraída (AVE) y el alfa de Cronbach (CA) como criterios de validación (Cronbach, 1951). AVE intenta medir la cantidad de varianza que una componente de la variable latente captura de su indicador respecto a la cantidad debida al error de medida. Deben ser mayores que las correlaciones al cuadrado. Se recomienda que esa cantidad sea mayor del 0,5 para que los indicadores expliquen más que el error, pero si ese valor se encuentra alrededor de 0,3 también se pueden aceptar, si está justificado. CA mide la consistencia interna del cuestionario. Se basa en el análisis de las correlaciones promedios entre los ítems referidos a un mismo aspecto, a partir de una sola administración del cuestionario. Se considera que es adecuado cuando es mayor de 0,6 (Nunnally y Bernstein, 1994).

Al no exigir la normalidad de las variables que definen los constructos a la hora de hacer la estimación, la ley de distribución de los estimadores obtenidos no es conocida. Por ello, para contrastar si los parámetros son significativos se hace uso de una técnica de bootstrapping. Esta consiste en crear $\mathrm{N}$ muestras similares a la obtenida con esa misma distribución empírica. A partir de cada una de ellas se obtendrá un estimador PLS diferente. Suponiendo que el promedio de todos ellos sigue aproximadamente una distribución normal, se evalúa su fiabilidad y valor (Efron, 1979). El proceso de estimación del modelo presenta varias fases. En primer lugar, se debe comprobar si los ítems utilizados se ajustan a los constructos definidos. Esta comprobación se compone de dos elementos: por una parte, se debe determinar si el efecto de cada uno de esos ítems sobre el constructo es significativo, es decir, si su efecto es realmente diferente de cero, lo que se comprobará con los t-estadísticos construidos por bootstrapping; por otra, se debe evaluar de modo conjunto si el constructo representa bien la información suministrada por los diferentes ítems, para lo cual haremos uso del AVE y del CA. Cuando alguno de los ítems que intervienen en el modelo no es significativo, se elimina del constructo y se rehacen los cálculos sin ese ítem. Una vez aceptada la validez de los constructos, se debe evaluar si el efecto entre ellos es significativo. Esta segunda fase debe llevarse a cabo mediante t-estadísticos calculados también por técnicas bootstrapping.

Para evaluar esos efectos, se hace uso de una metodología basada en trayectorias. Dicho proceso tiene varios elementos. En el nivel inicial se analiza el efecto de todos los constructos que actúan como variables explicativas sobre el constructo respuesta. Si en algún caso, esa relación no es significativa, se elimina de ese nivel. Los constructos que se mantienen en el modelo pasan a ser variables respuesta del siguiente nivel de trayectoria. A continuación, se repite el proceso hasta que no haya variables significativas en el modelo. 
Para el proceso de análisis, depuración y tratamiento de los datos, determinación de factores y evaluación de efectos se utilizaron los paquetes estadísticos SPSS (versión 15) y SMART-PLS (versión 2M3) (Ringle et al., 2005).

\section{RESULTADOS}

\section{Capital intelectual y ventajas competitivas}

Los resultados de aplicar el modelo anterior a los datos de las empresas madereras de Oberá confirman la composición de los constructos relativos al capital relacional, estructural y humano, al igual que al resto de elementos introducidos en el modelo, puesto que los ítems utilizados para definirlos eran todos significativos mediante los test bootstrap salvo los resultados empresariales, puesto que dicho constructo no incluía de modo significativo ni los costos, ni los beneficios, por lo que fueron eliminados del estudio, al igual que se aceptaba el modelo global (Fernández-Jardón y Martos, 2016).

Los efectos de las relaciones entre constructos vienen recogidos en la Tabla . Tal como mostraron Fernández-Jardón y Martos, (2016), el efecto principal sobre los resultados empresariales era debido a los factores estratégicos, que eran afectados por el territorio y las capacidades organizativas. Estas estaban constituidas por los recursos tangibles y el capital intelectual.

Tabla 1. Efectos entre constructos

\begin{tabular}{cccccc}
\hline Constructos & $\begin{array}{c}\text { Original } \\
\text { Sample }\end{array}$ & $\begin{array}{c}\text { Sample } \\
\text { Mean }\end{array}$ & $\begin{array}{c}\text { Standard } \\
\text { Deviation }\end{array}$ & $\begin{array}{c}\text { Standard } \\
\text { Error }\end{array}$ & $\begin{array}{c}\text { Test } \\
\text { Statistics }\end{array}$ \\
\hline HC -> CE & 0,76 & 0,77 & 0,05 & 0,05 & 14,44 \\
CE -> CR & 0,70 & 0,71 & 0,04 & 0,04 & 17,06 \\
CR -> CO & 0,66 & 0,67 & 0,08 & 0,08 & 8,53 \\
RT -> CO & 0,33 & 0,32 & 0,08 & 0,08 & 3,94 \\
Zona -> FE & 0,38 & 0,38 & 0,09 & 0,09 & 4,13 \\
CO -> FE & 0,47 & 0,49 & 0,07 & 0,07 & 6,42 \\
FE -> RE & 0,42 & 0,49 & 0,06 & 0,06 & 6,70 \\
\hline
\end{tabular}

HC: capital humano; CE: capital estructural; CR: capital relacional; CO:

competencias organizativas; RT: recursos tangibles; Zona: territorio; FE: factores estratégicos; RE: resultados empresariales. Fuente: Elaboración propia

Nuestro interés se centra en analizar separadamente cada una de las dimensiones del capital intelectual. Se comprobó que solo el capital relacional tiene efectos significativos directos sobre las capacidades organizativas. Este, a su vez, está afectado por el capital estructural. Finalmente, el capital humano afecta al capital estructural, estando en la raíz de todo el proceso (Bontis et al., 2000). Por tanto, se puede decir que todas las componentes del capital intelectual afectan a las capacidades organizativas, si bien no todas directamente.

En ese sentido, deben establecerse prioridades en las estrategias de diseño de capital intelectual en las pymes, puesto que su comportamiento y sus efectos son diferenciables.

\section{CONCLUSIONES Y DISCUSION}

Coincidiendo con los estudios recientes se puede afirmar que el capital intelectual es un factor de competitividad de la empresa y, en particular, para las pymes, tanto en países desarrollados (Vergauwen et al., 2007) como en países en vías de desarrollo (Bontis et al., 2000; Fernández-Jardon y Martos, 
2009). Este trabajo analiza su efecto dentro de un modelo de ventajas competitivas (Kamukama et al., 2011), de forma que se puedan evaluar los factores y el proceso mediante el cual los diferentes elementos del capital intelectual afectan a los resultados de la empresa. Complementariamente, este trabajo tiene en cuenta las características específicas de las pymes de Latinoamérica, que presentan matices en los resultados generalmente obtenidos en este tópico.

Las pymes latinoamericanas partieron de una situación muy inferior al estándar de los países desarrollados en gestión de negocios, organización productiva y relaciones laborales. Por ello, han tenido que realizar un gran esfuerzo de aprendizaje y de adaptación técnica para compensar esas diferencias (Quintar y Castaño, 1992). En particular, las limitaciones territoriales incrementan ese esfuerzo. En consecuencia, estas pymes presentan características propias, por lo que la obtención de ventajas competitivas presenta algunas diferencias respecto a los países desarrollados. Los empresarios mejoran sus factores estratégicos a partir de las capacidades organizativas y del territorio y, a través de ellos, se generan mejores resultados en la empresa. Las proposiciones resaltan la importancia del capital intelectual, lo que coincide con trabajos en países desarrollados (Vergauwen, 2007).

Este trabajo presenta un modelo de ventajas competitivas apoyado en el capital intelectual. Siguiendo ese modelo, se ha contrastado que el capital intelectual es fuente de ventajas competitivas en empresas del clúster de la madera de Oberá (Argentina). Para ello se ha comprobado, mediante técnicas PLS, si las componentes del capital intelectual intervienen entre los recursos que actúan sobre las capacidades organizativas. Las conclusiones sugieren aspectos particulares que, con la debida cautela, pueden ser generalizados a otras pymes similares. El capital humano aparece como base del capital intelectual. Por lo tanto, se deben promover actividades que potencien la actitud del personal de la empresa (Coff y Kryscynski, 2011). De modo semejante, sería conveniente fomentar la formación de los directivos y trabajadores, necesaria para que las pymes se adapten a la competitividad internacional y, en esa línea, se necesita elaborar políticas que den apoyo a esa actividad (Labarca, 1999). Finalmente, es necesario facilitar las diferentes capacidades del personal de la empresa adaptadas a la estrategia de la compañía (Ployhart y Moliterno, 2011). Esos aspectos ayudan a mejorar la cultura, la tecnología y la organización de las pymes, lo cual facilita la creación de redes de cooperación y relaciones más estables y duraderas entre los clientes y proveedores, lo que permitiría superar el individualismo existente. Esas relaciones favorecen que los procesos y sistemas sean más elaborados, constituyendo capacidades organizativas que llevan a las pymes a ser más competitivas.

Por tanto, estas pymes hacen uso del capital intelectual como factor de competitividad, pero no lo utilizan para constituir competencias distintivas que les permitan mantener su éxito empresarial en el largo plazo, sino que únicamente sirve para potenciar los factores estratégicos a través de las capacidades organizativas, de modo que se mantenga su competitividad en el mediano plazo (Gatto, 1999). Una utilización más estratégica del capital intelectual, exigiría un refuerzo de la formación, la profesionalización y la cooperación, elementos asociados al capital humano, al capital estructural y al capital relacional, respectivamente.

El capital humano afecta al capital estructural. Esto sugiere que las actividades de formación son cada vez más necesarias en estas empresas. Además, esa formación debe estar orientada hacia la gestión estratégica y la cualificación 
tecnológica de los recursos humanos para conseguir que las empresas sean competitivas ante el reto de la globalización. El hecho de que el capital humano afecte a los resultados empresariales, a través de los factores estratégicos, señala la importancia de alinear los recursos humanos con la estrategia de la empresa (Wright y McMahan, 2011).

El capital estructural es también un recurso esencial para competir. Por ejemplo, (Dixon, 1992) sugiere que es necesario el aprendizaje organizativo y Torrent-Sellens y Ficapal Cusí (2010), el conocimiento tecnológico de sus trabajadores. Paralelamente, es necesaria una mayor exigencia en la profesionalización y modernización de las empresas, que les permita mejorar su capital estructural, reforzando su cultura (Barney, 1996) con una mayor orientación al mercado (Fernández-Jardon et al., 2007) y con una gestión más eficiente (Gatto, 1999).

Una consecuencia de la evolución histórica de las pymes latinoamericanas es su carácter fuertemente individual e interior a la empresa de los procesos de aprendizaje, que ha dado lugar a experiencias muy heterogéneas, con una fuerte especificidad de las respuestas, desaprovechando las posibilidades de sinergias y de articulación productiva y el beneficio de economías externas (Quintar y Castaño, 1992). Por ese motivo, aunque las acciones individuales de las empresas pueden mejorar su rentabilidad y su posición competitiva, esta se sustentará más sólidamente, si las empresas se integran y comparten un tejido industrial y empresarial que desarrolle ventajas competitivas sistémicas de carácter colectivo. De lo contrario, las posibilidades de reconstruir y ampliar las capacidades básicas estarán restringidas muy fuertemente por los límites propios de la empresa (Gatto, 1999). En consecuencia, se deben elaborar redes de cooperación y fortalecer los conglomerados productivos en los que se integren las pymes, de modo que se potencie la generación de valor para el cliente final y no solo la satisfacción de la demanda actual.

Los resultados empíricos han sido evaluados en un caso particular, por lo que deben tomarse con cautela las conclusiones antes de generalizarlas a otras pymes. No obstante, proponen ideas que pueden ser contrastadas con otros estudios.

Schmitz y Nadvi (1999) sugieren que las políticas deben adecuarse a las necesidades específicas de cada tipología de clúster, puesto que sus reacciones y sus modos de crecimiento son muy diferentes. En ese sentido, las proposiciones presentadas para las pymes basadas en recursos naturales permitirán elaborar políticas adecuadas que potencien su competitividad y un desarrollo sostenible de los territorios donde realizan su actividad (Gatto, 1999).

Los aspectos relativos al conocimiento del funcionamiento estratégico de estas pymes van a proveerlas de instrumentos para reflexionar sobre la estrategia que siguen y adaptarla a los avances del conocimiento, dándoles a conocer los factores que potencian su competitividad y les permitan mejorar, de modo que faciliten la creación de riqueza y empleo.

\section{REFERENCIAS BIBLIOGRÁFICAS}

Barney, J.B. (1996), "The Resource-based Theory of the Firm", Organization Science, INFORMS: Institute for Operations Research, Vol. 7 No. 5, p. 469.

Bercovich, N., Katz, J.M. and United Nations. Economic Commission for Latin America and the Caribbean. (2003), El Desarrollo de Complejos Forestales En América Latina , CEPAL.

Bontis, N. (2001), "Assessing knowledge assets: a review of the models used to measure intellectual capital", International Journal of Management Reviews, Vol. 3 No. 1, pp. 41-60. 
Bontis, N., Keow, W.C.C. and Richardson, S. (2000), "Intellectual capital and business performance in Malaysian industries", Journal of Intellectual Capital, MCB UP Ltd, Vol. 1 No. 1 , pp. $85-100$.

Camisón-Zornoza, C. (1997), La competitividad de la PYME industrial española: estrategia y competencias distintivas, Civitas, Madrid, available at: http://dialnet.unirioja.es/servlet/libro?codigo=190507 (accessed 16 January 2013).

Coff, R. y Kryscynski, D. (2011), "Drilling for Micro-Foundations of Human Capital-Based Competitive Advantages", Journal of Management, Vol. 37 No. 5, pp. 1429-1443.

Coff, R.W. (2008), " Teamwork as Competitive Advantage", in West, M.A., Tjosvold, D. and Smith, K.G. (Eds.), International Handbook of Organizational Teamwork and Cooperative Working, John Wiley \& Sons Ltd, Chichester, UK, pp. 483-499.

Dixon, N.M. (1992), "Organizational learning: A review of the literature with implications for HRD professionals", Human Resource Development Quarterly, Vol. 3 No. 1, pp. 2949.

Escobar, P. (2004), "La forestación se consolida como una alternativa productiva en Misiones", Argentina Forestal, available at: http://www.argentinaforestal.com/actualidad/politica-y-economia/24-general/65la-forestaci-e-consolida-como-una-alternativa-productiva-en-misiones (accessed 14 November 2006).

Fernández-Jardón, C.M. (2008), Capital Intelectual E Internacionalización de La Pyme Gallega, Universidade de Vigo, Vigo.

Fernández-Jardón, C.M. and Martos, M.S. (2016), "Capital intelectual y ventajas competitivas en pymes basadas en recursos naturales de Latinoam\{é\}rica", Innovar: Revista de Ciencias Administrativas $Y$ Sociales, Escuela de Administraci\{ó\}n de Empresas y Contadur\{'\i\}a Pública, Vol. 26 No. 60, pp. 117132.

F-Jardon, C. y Martos, M.S. (2011), "Un método para determinar competencias distintivas en pequeñas y medianas empresas?", Revista de Administração da UFSM, Vol. 4 No. 2, pp. 195-214.

F-Jardon, C.M.y Martos, M.S. (2009), "Intellectual capital and performance in wood industries of Argentina", Journal of Intellectual Capital, Vol. 10 No. 4, pp. 600 616.

Flöstrand, P. (2006), "The sell side - observations on intellectual capital indicators", Journal of Intellectual Capital, Emerald Group Publishing Limited, Vol. 7 No. 4, pp. 457473.

Gatto, F. (1999), "Mercosur: its challenges to small and medium-sized industrial enterprises in terms of competition", Cepal Review, No. 68, pp. 61-77.

Giuliani, E., Pietrobelli, C. and Rabellotti, R. (2005), "Upgrading in Global Value Chains: Lessons from Latin American Clusters", World Development, Vol. 33 No. 4, pp. 549-573.

Grant, R.M. (1991), "The Resource-Based Theory of Competitive Advantage: Implications for Strategy Formulation", California Management Review, Vol. 33 No. 3, pp. 114135.

Grant, R.M. (2005), Contemporary Strategy Analysis, Fifth., John Wiley \& Sons, New York.

Helfat, C.E. y Lieberman, M. (2002), "The birth of capabilities: market entry and the importance of pre-history", Industrial and Corporate Change, Vol. 11 No. 4, pp. $725-760$.

Humble, J., Jackson, D. and Thomson, A. (1994), "The strategic power of corporate values", Long Range Planning, Vol. 27 No. 6, pp. 28-42.

INDEC. (2002), "Censo Nacional", Instituto Nacional de Estadistica Y Censos de Argentina, available at: http://www.indec.mecon.ar (accessed 4 November 2005).

Jardon, C.M., Gutawski, S., Martos, S., Aguilar, C. and Barajas, A. (2007), Visión Estratégica de La Cadena Empresarial de La Madera de Oberá, Misiones, EDUNAM Editorial Universitaria de la Universidad Nacional de Misiones, Posadas.

Kamoche, K. (1996), "Strategic Human Resource Management within a Resource-Capability View of the Firm", Journal of Management Studies, Vol. 33 No. 2, pp. 213-233.

Kamukama, N., Ahiauzu, A. and Ntayi, J.M. (2011), "Competitive advantage: mediator of intellectual capital and performance", Journal of Intellectual Capital, Vol. 12 No. 1, pp. $152-164$. 
Kantis, H. (1996), Inercia e innovación en las conductas estratégicas de las PYMES Argentinas. Elementos conceptuales y evidencias empíricas., No. 73, Buenos Aires, available at: http://www.cepal.org/cgibin/getProd.asp?xml=/publicaciones/xml/2/4222/P4222.xml\&xsl=/argentina/tpl/p9f .xsl\&base =/argentina/tpl/top-bottom.xsl (accessed 16 January 2013).

Labarca, G. (1999), "Capacitación en pequeñas empresas en América Latina", Revista de La CEPAL, Vol. 67 No. 1, pp. 33-48.

Lawler, E.E. (2009), "Make Human Capital a Source of Competitive Advantage", Organizational Dynamics, Vol. 38 No. 1, pp. 1-7.

Martín-de-Castro, G., Delgado-Verde, M., López-Sáez, P. and Navas-López, J.E. (2010), "Towards 'An Intellectual Capital-Based View of the Firm': Origins and Nature", Journal of Business Ethics, Vol. 98 No. 4, pp. 649-662.

Martín-de-Castro, G., Navas-López, J.E., López-Sáez, P. and Alama-Salazar, E. (2006), "Organizational capital as competitive advantage of the firm", Journal of Intellectual Capital, Vol. 7 No. 3, pp. 324-337.

Maslatón, C. y Ladrón Gonzalez, A. (2008), "Cifras para pensar: estructura productiva de la industria maderera", Argentina Forestal .com, Vol. 4 No. 58, pp. 14-16.

Milesi, D., Moori, V., Robert, V. and Yoguel, G. (2007), "Desarrollo de ventajas competitivas: pymes exportadoras exitosas en Argentina, Chile y Colombia", CEPAL Review, No. 92, pp. 25-43.

Newbold, P., Carlson, W.L. and Thorne, B.M. (2002), Statistics of Business and Economic, 5 th., Prentice Hall, New York.

Nunnally, J. y Bernstein, I. (1994), Psychometric Theory, 3th ed., McGraw-Hill Humanities/Social Sciences/Languages, New York.

O'Donnell, A., Gilmore, A., Carson, D. and Cummins, D. (2002), "Competitive advantage in small to medium-sized enterprises", Journal of Strategic Marketing, Routledge, Vol. 10 No. 3, pp. 205-223.

Pelham, A. (1997), "Mediating Influences on the Relationship Between Market Orientation and Profitability in Small Industrial Firms", Journal of Marketing Theory and Practice, Vol. 5 No. 3, pp. 55-76.

Ployhart, R.E. y Moliterno, T.P. (2011), "Emergence of The Human Capital Resource: A Multilevel Model", Academy of Management Review, Academy of Management, Vol. 36 No. 1 , pp. $127-150$.

Porter, M.E. (1985), Competitive Advantage, The Free Press, New York.

Prahalad, C.K. y Hamel, G. (1990), "The Core Competence of the Corporation", Harvard Business Review, Springer, Vol. 68 No. 3, pp. 79-91.

Quintar, A. y Castaño, A. (1992), Historia de Las Pequeñas Y Medianas Empresas Metalmecánicas de Rosario, Programa CFI-CEPAL, Buenos Aires.

Reed, K.K., Lubatkin, M. and Srinivasan, N. (2006), "Proposing and Testing an Intellectual Capital-Based View of the Firm", Journal of Management Studies, Vol. 43 No. 4, pp. 867-893.

Ringle, C.M., Wende, S. and Will, A. (2005), "SmartPLS Release: 2.0 (beta)", SmartPLS Internet, Hamburg, available at: http://www.smartpls.de.

Roos, G., Roos, J., Dragonetti, N.C. and Edvinsson, L. (1997), Intellectual Capital: Navigating in the New Business Landscape, New York University Press, New York, available

at: http://www.iberlibro.com/servlet/BookDetailsPL?bi=10200721492\&searchurl=an $=j$ ohan +roos +goran +roos + nicola $+c+$ dragonetti+leif + edvinsson \&bsi $=0 \& d s=30$ (accessed 16 July 2013).

Schmitz, H. and Nadvi, K. (1999), "Clustering and Industrialization: Introduction", World Development, Vol. 27 No. 9, pp. 1503-1514.

Spencer, L.M. and Spencer, S.M. (1993), Competence at Work: Models for Superior Performance, Wiley, New York.

Toledo-López, A., Díaz-Pichardo, R., Jiménez-Castañeda, J.C. and Sánchez-Medina, P.S. (2012), "Defining success in subsistence businesses", Journal of Business Research, Vol. 65 No. 12, pp. $1658-1664$.

Torrent-Sellens, J. and Ficapal Cusí, P. (2010), "TIC, co-innovación y productividad empresarial: evidencia empírica para Cataluña y comparación internacional de resultados.", Revista de Economía Mundial, Vol. 47-48 No. 26, pp. 203-233. 
Tsai, K.-H. (2004), "The impact of technological capability on firm performance in Taiwan's electronics industry", The Journal of High Technology Management Research, Vol. 15 No. 2, pp. 183-195.

Vergauwen, P., Bollen, L. and Oirbans, E. (2007), "Intellectual capital disclosure and intangible value drivers: an empirical study", Management Decision, Vol. 45 No. 7, p. 1163.

Wang, W.-Y. and Chang, C. (2005), "Intellectual capital and performance in causal models: Evidence from the information technology industry in Taiwan", Journal of Intellectual Capital, Vol. 6 No. 2, pp. 222-236.

Welbourne, T.M. and Pardo del Val, M. (2008), Relational Capital: Strategic Advantage for Small and Medium-Size Enterprises (SMES) Negotiation and Collaboration, No. CEO PUBLICATION T 08-13 (549), Business Management, Vol. 1, L o s A n g e I e s, available at: http://ceo.usc.edu/pdf/t08_13.pdf.

Wernerfelt, B. (1984), "A resource-based view of the firm", Strategic Management Journal, John Wiley \& Sons, Vol. 5 No. 2, pp. 171-180.

Wilkinson, A. (1999), "Employment relations in SMEs", Employee Relations, MCB UP Ltd, Vol. 21 No. 3, pp. 206-217.

Wright, P.M. and McMahan, G.C. (2011), "Exploring human capital: putting 'human' back into strategic human resource management", Human Resource Management Journal, Vol. 21 No. 2, pp. 93-104.

Yang, J. (2008), "The determinants of supply chain alliance performance: an empirical study", http://dx.doi.org/10.1080/00207540701376358, Taylor \& Francis Group .

Yoguel, G. (2000), "Creating capabilities in local environments and production networks", CEPAL Review, No. 71, pp. 101-115.

Zevallos, E. V. (2006), "Obstáculos al desarrollo de las pequeñas y medianas empresas en América latina", Cuadernos de Difusión, Vol. 11 No. 20, pp. 75-97. 